\title{
Taking quartic roots of 2
}

\author{
Thinh D. NguYeN* \\ Moscow State University \\ kosmofarmer@gmail.com \\ July 22, 2018
}

\begin{abstract}
Taking square roots of -1 modulo composite numbers was shown to be hard in our previous work [5]. This short note only discusses the same phenomenon of taking quartic roots of 2 modulo prime numbers. Diligent readers of Prasolov and I. F. Sharygin can realize how effective each problem, each chapter helps shape our mathematical reasoning.
\end{abstract}

\section{BACKGROUND, NOTATION AND CLAIMS}

While working with modular polynomials, nothing can be more interesting and also striking than raising the degree of them. Previously, we discussed the equation $x^{2} \equiv-1 \bmod n$ where $n$ can be any natural number. Now, we want to deal with quartic equation of the form $x^{4} \equiv 2 \bmod p$ where $p$ is a prime number. The long story of this equation and variants has begun since 1749. Around that time, Euler conjectured the necessary and sufficient condition of $p$ for the existence of a quartic root of 2 modulo $p$. His book Tractatus de numeroroum doctrina capita sedecim quae supersunt [1] was published in 1849, one hundred years after he wrote it. In this book, he conjectured that 2 is a fourth power residue $(\bmod p)$ iff. $p$ is of the form $x^{2}+64 y^{2}$. For example, 2 is a fourth power residue (mod 73), since $73=3^{2}+64$.

Gauss proved this conjecture in 1828 in his first monograph [2] on biquadratic reciprocity. Historically, Euler's conjecture was widespread among mathematicians long before officially printed. Together with Fermat's $4 n+1$ theorem, we can see that such a prime $p$ can be uniquely represented as $a^{2}+b^{2}$, up to the swapping of $a$ and $b$. Parity of $a$ and $b$ must be different as well. So, Gauss's

*Perebor 
condition can be stated as that the even one among $a$ and $b$ must be divisible by 8 . H. Davenport wrote an excellent book [3] on arithmetics, which contains, in much details, various topics.

Modern mathematics has since developed so much fruitful ideas to solve polynomials both for real roots and modular roots. Zhi-Hong Sun [4] extends the condition for other values of natural number $m \neq 2$ to be quartic residue mod $p$.

Gauss's condition can be seen as a polynomial-time reduction between computational problems. Some definitions are necessary.

\section{i. Definitions}

Quartic-2-Modulus $=\{p \mid p$ is congruent to 1 modulo 4 and 2 is a quartic residue $\bmod p\}$

Feasible-Quad-Diophantine $=\left\{<n, d>\mid\right.$ the Diophantine equation $x^{2}+d y^{2}=n$ is feasible\}

Property-of-Diophantine-Solution $=\left\{n \mid\right.$ the Diophantine equation $x^{2}+y^{2}=n$ is satisfied by a solution $(a, b)$ where $a$ or $b$ is divisible by 8$\}$

So, now the following claims are immediate from Gauss's condition.

\section{ii. Claims}

Claim 1: Quartic-2-Modulus $\leq_{p}$ Feasible-Quad-Diophantine

Claim 2: Quartic-2-Modulus $\leq_{p}$ Property-OF-Diophantine-Solution

If you have the ability to solve Diophantine equation, you have enough capabilities to deal with much of modular arithmetics. Even an algorithm to account for the existence of a solution to Diophantine equation is of vastly achievements.

\section{REFERENCES}

[1] Euler, Leonhard (1849), Tractatus de numeroroum doctrina capita sedecim quae supersunt, Comment. Arithmet. 2

[2] Gauss, Carl Friedrich (1828), Theoria residuorum biquadraticorum, Commentatio prima, GÃúttingen: Comment. Soc. regiae sci, GÃúttingen 6

[3] H. Davenport, The Higher Arithmetic: An Introduction to the Theory of Numbers, Cambridge University Press 
[4] Zhi-Hong Sun, Quartic residues and binary quadratic forms, Journal of Number theory 113 pp.10-52, Elsevier, 2005

[5] Thinh D. Nguyen, Taking square roots of -1 , https://osf.io/5fmhd/, 2018 